\title{
Circular-Navigation-Guidance Law for Precision Missile/Target Engagements
}

\author{
Ian R. Manchester* and Andrey V. Savkin ${ }^{\dagger}$ \\ University of New South Wales, Sydney, New South Wales 2052, Australia
}

\begin{abstract}
A new precision guidance law with impact angle constraint for a two-dimensional planar intercept is presented. It is based on the principle of following a circular arc to the target, hence the name circular navigation guidance. The guidance law does not require range-to-target information. We prove that it attains a perfect intercept under certain ideal conditions. In a broader range of conditions, it is shown to perform favorably when compared to another law from the literature.
\end{abstract}

\section{Introduction}

A LMOST all guidance laws discussed in the literature have one objective: to reduce to zero the distance between the missile and the target. This is not always sufficient; in some cases the direction from which the missile approaches the target is also important. For example, it might be necessary to disable an aircraft without hitting a dangerous payload or the pilot, and many heavily armoured targets are much more susceptible from certain angles. Despite the many applications and recent interest in such a guidance law, there has been comparatively little research activity. In this paper we present a simple but effective new guidance law with an impact angle constraint.

The first published work on this problem was by Kim and Grider, ${ }^{1}$ and considers the guidance of a ballistic reentry vehicle. York and Pastrick $^{2}$ extended this work by including a first-order autopilot. More recent work ${ }^{3}$ has focused on moving and maneuvering work by introducing a time-varying bias term to the proven proportionalnavigation-guidance (PNG) law (for example, see Chapters 2-6 of Refs. 4 and 5 for more advanced laws without impact angle constraints), which was found to be successful over a certain range of angles. Optimal control theory was used to derive another law in Ref. 6 for the case in which missile velocity varies as a result of aerodynamic effects. The authors' simulations showed this law to be successful against a slow-moving target, such as a ship, providing assumptions such as small initial heading error are satisfied. In Ref. 7 a time-optimal control scheme is formulated for impact angle control against a stationary target, such as a building. This work made use of numerical integration techniques to choose an openloop control satisfying the guidance goal. In Ref. 8 linear quadratic regulator and $H^{\infty}$ formulations are shown to be applicable to this problem. However, these rely on a linearized system allowing acceleration of the missile in any direction, which is not possible in most existing missile systems. In this paper we consider more practical restrictions resulting in a nonlinear model.

The new law presented here is based on the simple geometrical principle of following a circular arc toward the target. This theoretically guarantees zero miss distance and perfect impact angle under certain reasonable conditions. A significant advantage of our guidance law is that, unlike all other solutions to this problem of which the authors are aware, knowledge of the range to the target is not required.

Received 7 September 2004; revision received 12 April 2005; accepted for publication 24 April 2005. Copyright (C) 2005 by the American Institute of Aeronautics and Astronautics, Inc. All rights reserved. Copies of this paper may be made for personal or internal use, on condition that the copier pay the $\$ 10.00$ per-copy fee to the Copyright Clearance Center, Inc., 222 Rosewood Drive, Danvers, MA 01923; include the code 0731-5090/06 $\$ 10.00$ in correspondence with the CCC.

*Research Student, Electrical Engineering; ianm@student.unsw.edu.au. Student Member AIAA.

${ }^{\dagger}$ Professor of Electrical Engineering.
Aerodynamic effects such as drag are neglected, and both target and missile are assumed be mathematical points moving in a twodimensional plane with constant speed. This means that the control input (and the target's evasive maneuver) can only cause acceleration perpendicular to the missile's (or target's) velocity, that is, perform a turning motion.

In this paper we did not consider issues of measurement noise or modelling error. In another paper, ${ }^{9}$ an earlier version of the guidance law here described was examined in conjunction with $H^{\infty}$-like robust control law (see Ref. 10) and a robust extended Kalman filter (see Refs. 11 and 12).

The structure of the paper is as follows: first we describe the dynamic model and introduce all relevant variables. Then the problem statement is given mathematically in terms of these, and the information available to the guidance law is discussed. Next is a short section in which we provide the equations for the guidance law. Following this, the reasoning behind this law and a few mathematical results about it are discussed in some detail. And finally the guidance law is compared to another law from the literature with simulation studies, and some brief conclusions are given.

\section{System Description and Problem Statement}

The problem being considered is to reduce the miss distance as close as possible to zero and have the missile approach the target from as close as possible to some specified angle, which might be relative to the target's heading, or independent of it.

It is perhaps clearest to first present the system model in a Cartesian state space and from these states define some meaningful scalar and angular values that are used to formulate the guidance law.

We introduce three two-dimensional real vectors in Cartesian coordinates, which together fully describe the state of the system: 1) relative position $x_{R}(t):=$ target position-missile position; 2) missile velocity $v_{M}(t)$; and 3$)$ target velocity $v_{T}(t)$.

Now let a state vector $x(t)$ represent these combined:

$$
x(t):=\left[\begin{array}{c}
x_{1}(t) \\
\vdots \\
x_{6}(t)
\end{array}\right]:=\left[\begin{array}{c}
x_{R}(t) \\
v_{M}(t) \\
v_{T}(t)
\end{array}\right] \in \mathbb{R}^{6}
$$

System dynamics are defined in terms of a nonlinear state-space model,

$$
\dot{x}(t)=A x(t)+B_{1}(x) u_{c}(t)+B_{2}(x) a_{T}(t)
$$

where $u_{c}(t)$ is the control input, a one-dimensional acceleration issued perpendicular to the missile's current velocity vector. Similarly, $a_{T}(t)$ is a one-dimensional acceleration issued perpendicular to the target's velocity, if the target is maneuvering.

Using a simple model of Newtonian physics, and the acceleration restrictions just described, we obtain the following definition of the 


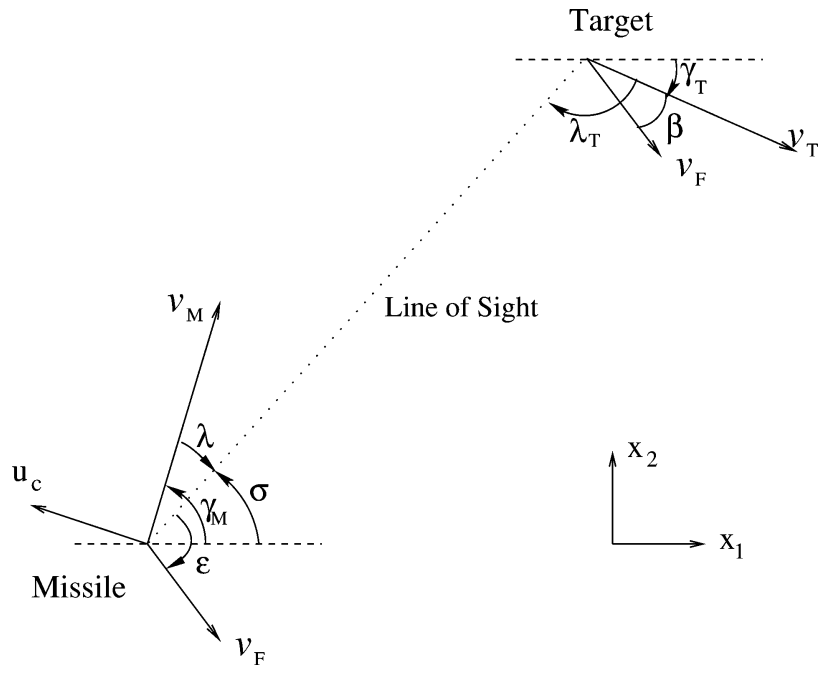

Fig. 1 Engagement geometry.

matrices in Eq. (1):

$$
\begin{gathered}
A=\left[\begin{array}{lll}
0_{2,2} & -I_{2} & I_{2} \\
& 0_{4,6}
\end{array}\right] \\
B_{1}(x)=\left[\begin{array}{c}
0_{2,1} \\
-x_{4}(t) / c_{M} \\
x_{3}(t) / c_{M} \\
0_{2,1}
\end{array}\right], \\
B_{2}(x)=\left[\begin{array}{c}
0_{4,1} \\
-x_{6}(t) / c_{T} \\
x_{5}(t) / c_{T}
\end{array}\right]
\end{gathered}
$$

where

$$
c_{M}:=\sqrt{x_{3}(t)^{2}+x_{4}(t)^{2}}, \quad c_{T}:=\sqrt{x_{5}(t)^{2}+x_{6}(t)^{2}}
$$

Furthermore, we define the following variables, which are visualized in Fig. 1:

$$
\begin{array}{rlrl}
r(t) & :=\sqrt{x_{1}(t)^{2}+x_{2}(t)^{2}}, & \sigma(t):=\tan ^{-1}\left[x_{2}(t) / x_{1}(t)\right] \\
\gamma_{M}(t):=\tan ^{-1}\left[x_{4}(t) / x_{3}(t)\right], & \gamma_{T}(t):=\tan ^{-1}\left[x_{6}(t) / x_{5}(t)\right]
\end{array}
$$

where the inverse tangent refers to the four-quadrant arctangent, mapping into the whole circle $(-\pi, \pi]$. In MATLAB ${ }^{\circledR}$, for example, the command ATAN2 achieves this. Whenever a numerical value for an angle is used in a calculation, it is the representative in the interval $(-\pi, \pi]$, which is meant.

Qualitatively, $r(t)$ is the range between missile and target, $c_{M}$ is the speed of the missile, and $c_{T}$ the speed of the target. Note that $c_{M}$ and $c_{T}$ are not time dependent: we are assuming that missile and target speeds are constant. The angle $\sigma(t)$ is that of the line of sight between missile and target, $\gamma_{M}(t)$ is the missiles heading angle, and $\gamma_{T}(t)$ is the target's heading angle. All are with respect to a horizontal reference, where a positive angle is one of anticlockwise rotation.

From these, we define two more angles used in circular navigation guidance $(\mathrm{CNG})$ :

$$
\lambda(t):=\sigma(t)-\gamma_{M}(t), \quad \lambda_{T}(t):=\pi+\sigma(t)-\gamma_{T}(t)
$$

That is, $\lambda(t)$ is the angle between the line-of-sight vector and the missile's velocity vector; $\lambda_{T}(t)$ is the equivalent value for the target.

We know to introduce the notion of approach-angle error. The idea is that, as the missile approaches the target immediately before impact, it should do so from a specific direction. The approach-angle error is the difference between the current approach angle $\sigma$ and that desired.
For the case where desired approach angle is defined relative to the target's velocity vector as $\gamma_{T}(t)+\beta$, the approach-angle error is

$$
\varepsilon(t):=\gamma_{T}(t)+\beta-\sigma(t)
$$

otherwise, the approach angle can be defined as an absolute bearing $\sigma^{*}$, independent of the target's motion, in which case it is

$$
\varepsilon(t):=\sigma^{*}-\sigma(t)
$$

\section{A. Information Patterns}

The information available to a guidance law is a crucial restriction in practice. We consider two different information patterns, labeled $\mathcal{T}_{1}$ and $\mathcal{T}_{2}$.

The first includes all information required for the perfect application of $\mathrm{CNG}$; the second is a reduced information set without information on target heading, which might be more appropriate in practice. In neither case do we assume that there is memory of past values, only the current values of the measurements are used:

1) $\mathcal{T}_{1}(t)$ : Knowledge of $\lambda(t), \dot{\lambda}(t), \lambda_{T}(t), c_{M}, c_{T}$, and either $\beta$, from which $\varepsilon$ can be derived, or $\varepsilon$ from which $\beta$ can be derived, depending on the whether or not impact angle is defined with respect to target heading.

2) $\mathcal{T}_{2}(t)$ : Knowledge only of $\lambda(t), \dot{\lambda}(t), \varepsilon(t), c_{M}$. That is, no knowledge of the target heading or speed. If $\beta$ is assigned, then for $\varepsilon(t)$ we again require knowledge of $\lambda_{T}(t)$. However in this case we emphasize the possibility of a desired impact angle independent of the targets heading.

Note that knowledge of the range $r(t)$ is not required in either information pattern. This can make our approach particularly useful when the sensor is a video camera or infrared sensor, from which range information is not typically available. Range could in theory be derived from $\mathcal{T}_{1}$ using the formula

$$
\dot{\lambda}=\left(c_{M} / r\right) \sin \lambda+\left(c_{T} / r\right) \sin \lambda_{T}-u_{c} / c_{M}
$$

so

$$
r=\frac{\left(c_{M} \sin \lambda+c_{T} \sin \lambda_{T}\right)}{\left(\dot{\lambda}+u_{c} / c_{M}\right)}
$$

However, in many practical circumstances (such as tail on approach) the denominator in the preceding equation will be zero or very small, making such calculations impossible or subject to very large errors.

\section{B. Problem Statement}

We now precisely state the problem we consider in this paper:

To define a function of the available information that will result in a control signal like so:

$$
u_{c}(t)=f\left[\mathcal{T}_{n}(t)\right] \in \mathbb{R}
$$

for $n \in\{1,2\}$, such that, for some unspecified final time $T$,

$$
\begin{gathered}
r(T) \rightarrow \min \\
|\varepsilon(T)| \rightarrow \min
\end{gathered}
$$

That is, range is minimized, and angle error is minimized.

\section{Guidance Law}

In this section we succintly state the exact formula we propose to solve the aforementioned problem. Its derivation, meaning, and resultant behavior are considered in the sequel.

First we note that with information patterns $\mathcal{T}_{1}$ and $\mathcal{T}_{2}$ we can measure $\dot{\lambda}$, from which we can easily compute $\dot{\sigma}$ :

$$
\dot{\sigma}(t)=\dot{\lambda}(t)+u_{c}(t) / c_{M}
$$

Note that if autopilot and airframe dynamics are taken into account, the actual missile acceleration should be used here in place of $u_{c}$. 
Let $k_{p}$ be a constant scalar gain, which is greater than zero. Two versions of the guidance law, corresponding to the information patterns just described, are proposed like so:

1) For information pattern $\mathcal{T}_{1}$,

$$
\begin{gathered}
\lambda_{\text {off }}:=\sin ^{-1}\left[\left(c_{T} / c_{M}\right) \sin \beta\right] \\
u_{c}(t)=2 c_{M} \dot{\sigma}(t)+k_{p}\left\{\lambda(t)-\left[\varepsilon(t)+\lambda_{\text {off }}\right]\right\}
\end{gathered}
$$

2) For information pattern $\mathcal{T}_{2}$,

$$
u_{c}(t)=2 c_{M} \dot{\sigma}(t)+k_{p}[\lambda(t)-\varepsilon(t)]
$$

\section{Guidance Law Derivation and Theoretical Results}

In this section we will present the reasoning behind the $\mathrm{CNG}$ guidance law and prove some theoretical results. Our explanation will come first to the simpler second and third forms of the law, from which the term circular navigation guidance was born, and then consideration of moving targets will lead us to the complete law.

First we define the following property of a target intercept:

Definition 1: A target intercept is said to be perfect if there exists some finite time $T$ such that

$$
r(T)=0, \quad \lim _{t \rightarrow T} \varepsilon(t)=0
$$

This definition essentially means that the missile hits the target, and the approach angle is exactly correct when when impact occurs. A limit is used in the second equation because if $r(T)=0$, then the angle $\sigma(T)$ is undefined, and therefore so is $\varepsilon(T)$.

Consider first the case of a stationary target, that is, $c_{T}=0$, $a_{T}(\cdot)=0$, and assume that the desired approach angle is arbitrarily defined because $\gamma_{T}()$ is undefined for a stationary target.

Our guidance law is based on the principle that two points, one of which has a tangent line defined, define a unique circle. The two points in question become the positions of the missile and the target, and the tangent line becomes the desired impact direction.

Theorem 1: Consider the case of a stationary target. Introduce the circle uniquely defined by the following properties: 1) the initial position of the missile lies on the circle, 2) the position of the target lies on the circle, and 3) the desired impact direction is a tangent to the target's position on the circle. Suppose that a controller of the form (4) is designed such that the angles $\lambda(t)$ and $\varepsilon(t)$ are kept exactly equal over the full flight time, then the missile's trajectory will be an arc on this circle. Furthermore, this will result in a perfect intercept, as defined in Definition 1.

Proof: In Fig. 2, suppose point $\mathrm{T}$ is the target position, point $\mathrm{M}$ is the missile position, and the line TV defines the desired impact

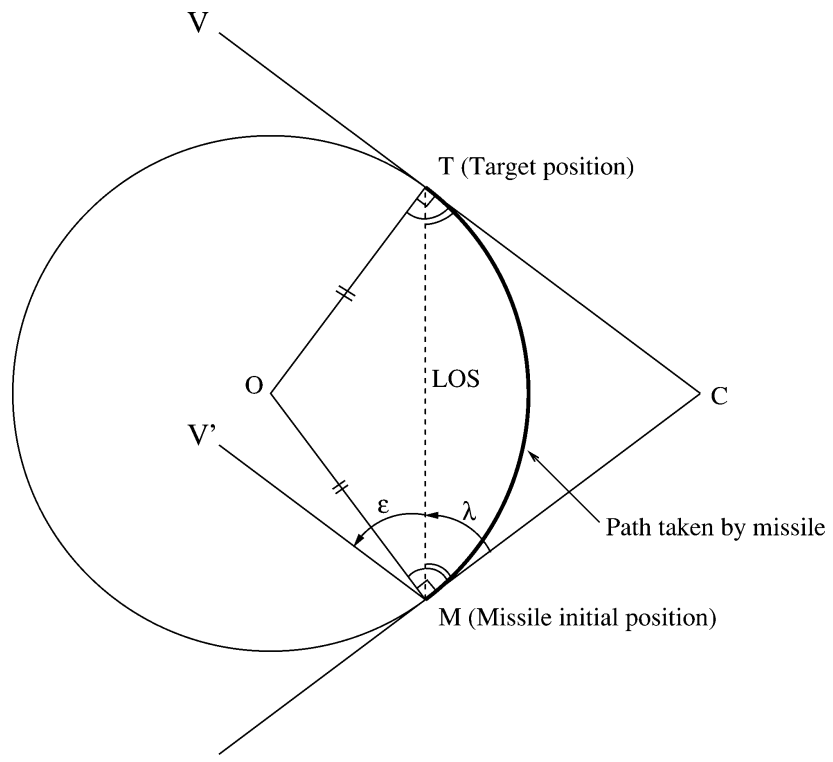

Fig. 2 Circular geometry for CNG with a stationary target. direction vector. The line MV' is parallel to TV. There is a unique circle passing through the points $\mathrm{M}$ and $\mathrm{T}$, with the tangent at $\mathrm{T}$ equal to the line TV.

Let $\mathrm{O}$ be the center of this circle, and $\mathrm{MC}$ be the tangent to the circle at the point $\mathrm{M}$, and $\mathrm{C}$ is the intersection of the two tangents. The angles $\lambda(t)=\angle C M T$ and $\varepsilon(t)=\angle T M V^{\prime}$ are shown.

$$
\begin{gathered}
\angle O M C=\angle O T C=\pi / 2 \quad \text { (circle tangent } \perp \text { radius) } \\
\angle C M T=\pi / 2-\angle O M T \quad \text { (complementary angles) } \\
\angle M T C=\pi / 2-\angle O T M \quad \text { (complementary angles) } \\
O T=O M \quad \text { (radii of a circle) } \\
\triangle O T M=\text { isosceles } \quad \text { (two sides equal) } \\
\angle O T M=\angle O M T \quad \text { (base angles, isosceles triangle) } \\
\angle C M T=\angle M T C \quad[\text { from Eqs. (10-12)] } \\
\angle M T C=\angle T M V^{\prime} \quad \text { (alternate angles, parallel lines) } \\
\angle C M T=\angle T M V^{\prime} \quad[\text { from Eqs. (13) and (14)] }
\end{gathered}
$$

Therefore, for any points $\mathrm{M}$ and $\mathrm{T}$, and any tangent line $\mathrm{TV}$, if $\lambda(t)=\varepsilon(t)$ for the entire intercept time, the missile will follow a circular path and impact at exactly the position $\mathrm{T}$ with a velocity the direction of TV.

This completes the proof of Theorem 1.

\section{A. Guidance Laws for a Stationary Target}

For the system with a stationary target, we have the following dynamical equations:

$$
\begin{gathered}
\dot{\sigma}(t)=\left[c_{M} / r(t)\right] \sin \lambda(t), \quad \dot{\lambda}(t)=\dot{\sigma}(t)-u_{c}(t) / c_{M} \\
\dot{\varepsilon}(t)=-\dot{\sigma}(t)
\end{gathered}
$$

Now suppose for a moment that the control signal is just $u_{c}(t)=2 c_{M} \dot{\sigma}$. It is clear that this makes $\dot{\lambda}(t)=\dot{\varepsilon}(t)$, so that if initial conditions satisfy $\lambda(0)=\varepsilon(0)$, then $\lambda(t)=\varepsilon(t)$ for all time the missile will describe a circular path to the target, and the intercept will be perfect.

If, however, there is some error in the initial conditions, say, $\lambda(0)=\varepsilon(0)+\Delta$, then the missile will describe a different circular path, and there will be an impact angle error of $\Delta$. The term involving $k_{p}$ is introduced to null such errors. With this term we have the guidance law (9), and defining $\Delta(t):=\lambda(t)-\varepsilon(t)$ we have

$$
\frac{\mathrm{d}}{\mathrm{dt}} \Delta(t)=-\frac{k_{p}}{c_{M}} \Delta(t)
$$

which decays exponentially with a time constant of $c_{M} / k_{p}$. The effect of the initial error $\Delta(0)$ on the final error $\Delta(T)$ can be driven as small as desired by increasing the gain $k_{p}$; this is clear when it is noted that in all cases $T \geq r(0) / c_{M}$, a straight line being the shortest path to the target, and so $\Delta(T) / \Delta(0) \leq \exp \left[-k_{p} r(0) / c_{M}^{2}\right]$. Note that the size of $k_{p}$ needed for a particular level of performance is not uniform in initial conditions.

Our method is fundamentally different to path-planning ideas common in the robotics literature, for which a particular trajectory is precomputed through space, and which the missile would then be regulated to. In our method, at every point in space, a different "desired circular path" exists.

This is illustrated in Fig. 3. The solid line is a simulated trajectory of the missile guided toward a stationary target, with a desired approach angle parallel to the vertical axis of the figure. At several points along the path, the desiredcircular path from that point is 


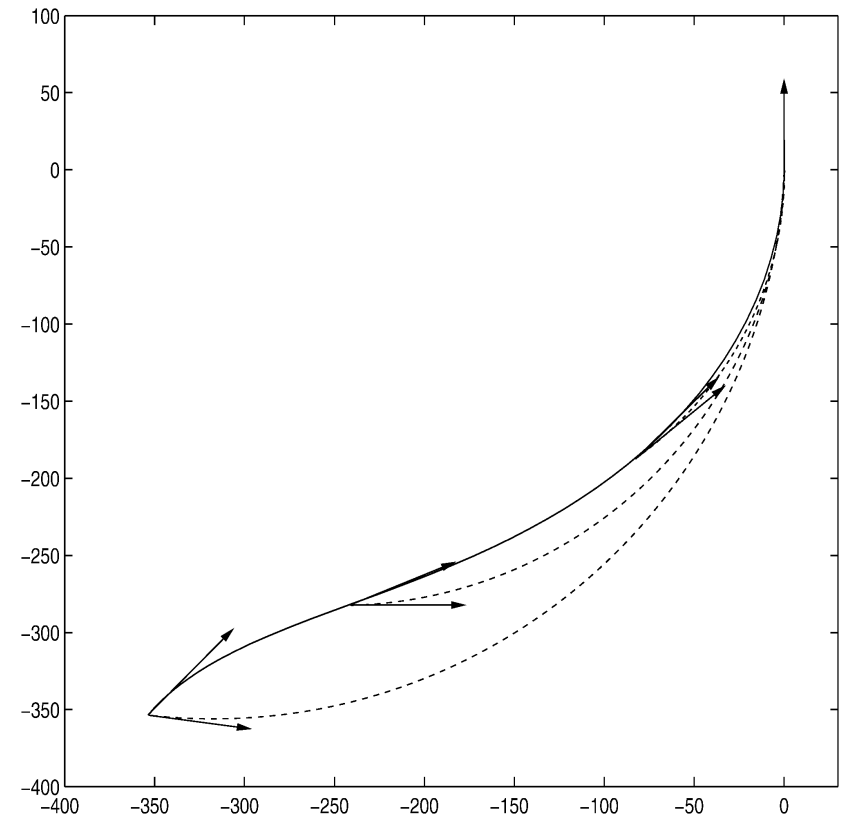

Fig. 3 Convergence to the circular path.

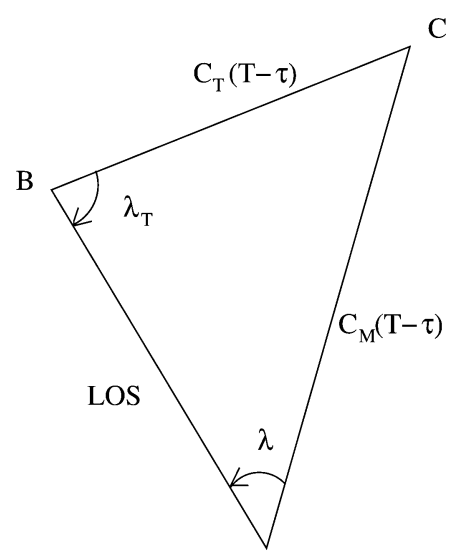

Fig. 4 Collision triangle.

A

shown with a dashed line. Tangent arrows are shown at these points to both the actual path taken, and the desired path, and the angle $\Delta(t)$ is the difference between these. It is clearly seen that $\Delta(t)$ converges toward zero (like a pair of scissors closing), but the actual path that the missile takes is entirely different to the first desired circular path.

Following this, one interpretation of the two parts of the guidance law is that the $2 c_{M} \dot{\sigma}(t)$ term makes the missile follow $a$ circular path, whereas the term $k_{p}[\lambda(t)-\varepsilon(t)]$ drives it toward the correct circular path.

\section{B. Guidance Law for a Moving Target}

In this section we consider a moving target. Doing so changes the dynamics of $\sigma, \lambda$, and $\varepsilon$ only slightly:

$$
\begin{gathered}
\dot{\sigma}(t)=\left[c_{M} / r(t)\right] \sin \lambda(t)+\left[c_{T} / r(t)\right] \sin \lambda_{T}(t) \\
\dot{\lambda}(t)=\dot{\sigma}(t)-u_{c}(t) / c_{M}, \quad \dot{\varepsilon}(t)=-\dot{\sigma}(t)
\end{gathered}
$$

and it is clear that the $\left(2 c_{M} \dot{\sigma}\right)$ part of the control law will have the same relevance.

We suppose for a moment that impact will occur (a fact that is proved in Theorem 2) and look at what happens in the final moments of flight, when the trajectory of the missile can be approximated as a straight line. The paths of missile and target will form a triangle like that in Fig. 4, which depicts the paths of missile A and target B,

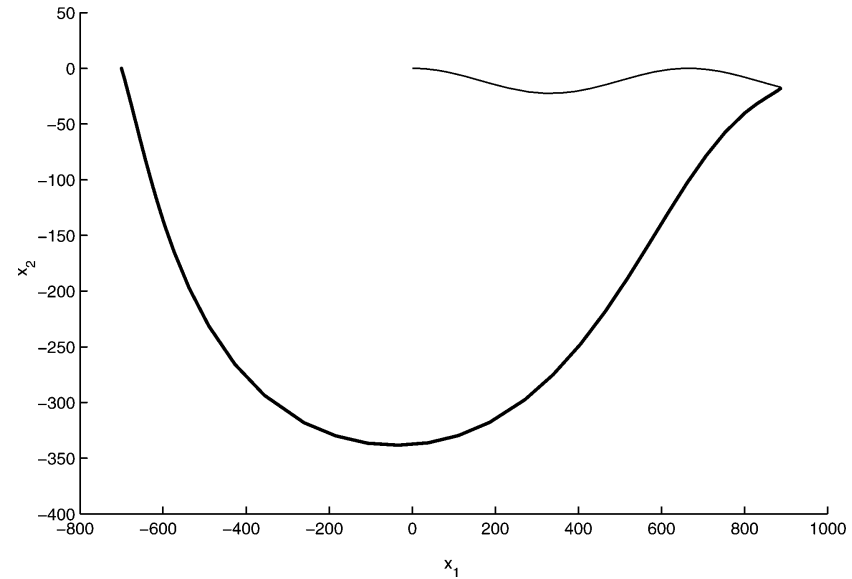

Fig. 5 Intercept of a maneuvering target.

the collision point $\mathrm{C}$, and the line of sight, a short time $\tau$ before collision. The angles $\sigma, \lambda, \lambda_{T}$ are constant over this period.

When $\sigma$ is constant, we have

$$
\left[c_{M} / r(t)\right] \sin \lambda=-\left[c_{T} / r(t)\right] \sin \lambda_{T}
$$

and if the impact is perfect, that is, $\varepsilon$ is zero, we should have $\lambda_{T}=\beta+\pi$, and consequently

$$
\lambda=\lambda_{\text {off }}:=\sin ^{-1}\left[\left(c_{T} / c_{M}\right) \sin \beta\right]
$$

Because the case $\varepsilon=0$ corresponds to the case $\lambda=\lambda_{\text {off }}$, we modify the strategy of keeping $\lambda(t)=\varepsilon(t)$ for all time to that of keeping $\lambda(t)=\varepsilon(t)+\lambda_{\text {off }}$ for all time.

The proportional part of the guidance law becomes $k_{p}\{\lambda(t)-$ $\left.\left[\varepsilon(t)+\lambda_{\text {off }}\right]\right\}$, and we have the first form of the guidance law, given in Eq. (8).

In Fig. 5 we show an example of an intercept using the first form of CNG against a target that is maneuvering, and so continuously changing $\gamma_{T}$, and perturbing $\varepsilon$. In the discussion of the preceding subsection, this amounts to an exogenous disturbance of the term $\Delta(t)$. We see that the missile follows roughly a circular path, but toward the end there is an inflexion in the trajectory, necessitated by the maneuvers of the target.

\section{Main Theoretical Results}

Broadly speaking, the theorem to follow states that everything we said about the second form of the guidance law in Sec. IV.A with a stationary target, that is, perfect intercept when initial conditions are perfect, arbitrarily small angle error when not, also holds for the first form of the control law with a moving target. This proof is slightly more complex and is contained in the following three subsections.

Theorem 2: Consider a target with constant nonzero velocity, but slower than the missile, that is, $c_{M}>c_{T}>0, a_{T}=0$, and suppose $\lambda(0)=\varepsilon(0)+\lambda_{\text {off. }}$ Then the guidance law given in Eq. (8) will result in a perfect intercept, as defined in definition 1 , for all initial conditions except those with $\varepsilon(0)=\pi$.

Two corollaries follow easily from this theorem. The first explains the results of using the second form of guidance law (9) against a moving target.

Corollary 1: If guidance law (9) is used, with information pattern $\mathcal{T}_{2}$, and $\lambda(0)=\varepsilon(0)$, then the missile will hit the target with zero miss distance and an angle error $\lim _{t \rightarrow T} \varepsilon(t)$ equal to $\lambda_{\text {off }}$.

Corollary 2: If initial conditions are not as desired, then angle error can be made arbitrarily close to zero for information pattern $\mathcal{T}_{1}$ and guidance law (8) and arbitrarily close to $\lambda_{\text {off }}$ for information pattern $\mathcal{T}_{2}$ and guidance law (9) by increasing the gain term $k_{p}$.

In Fig. 6 we have plotted the $\lambda_{\text {off }}$ vs $\beta$ for three values of $c_{T} / c_{M}$ : $0.5,0.3$, and 0.1 . Note the angle error goes to zero if $c_{T}=0$, which is the statement of Theorem 1 and also if $\beta=0$ or $\pi$. If the missile is much faster than the target, as is the case if the target is a land vehicle, then the angle error will be very small. 


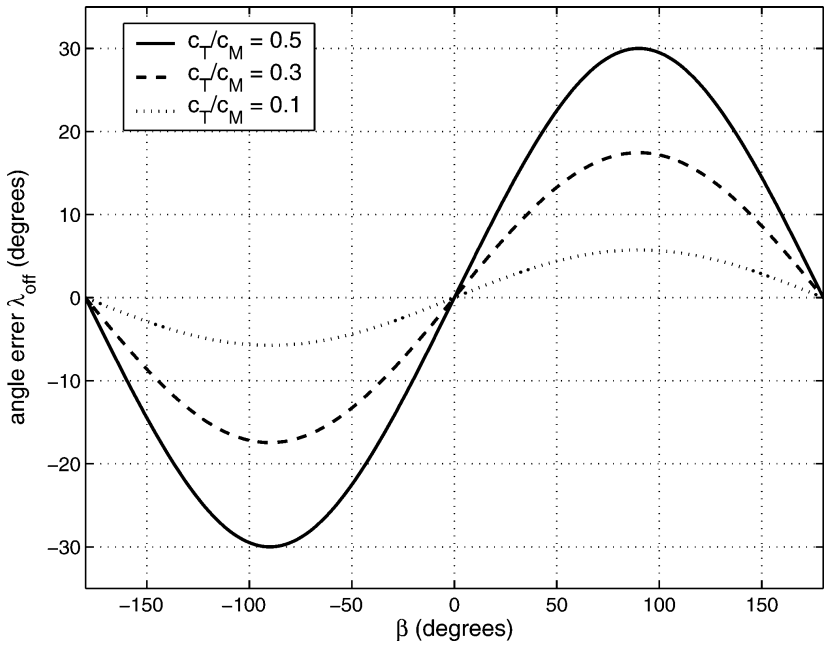

Fig. 6 Errors induced by using information patter $\mathcal{T}_{2}$. Angle error $\lambda_{\text {off }}$ vs desire approach angle $\beta$ for three values of $c_{T} / c_{M}$, the ratio of target speed to missile speed.

So if target velocity information is difficult to measure, but the missile is much faster than the target, then good performance will still be attained.

The proof of these results is presented the following three subsections.

\section{Convergence of Angle Error}

In this subsection we prove that the angle error converges to zero, more precisely: If $\lambda(t)=\varepsilon(t)+\lambda_{\text {off }}$ for all $t>0$, then the approach angle error $\varepsilon(t)$ converges to zero for all initial conditions except those with $\varepsilon(t)=\pi$.

Proof: We examine the dynamics of $\varepsilon$ :

$$
\dot{\varepsilon}=-\left(c_{M} / r\right) \sin \left(\varepsilon+\lambda_{\text {off }}\right)-\left(c_{T} / r\right) \sin \left(\lambda_{T}\right)
$$

Substituting for $\lambda_{\text {off }}$,

$$
\dot{\varepsilon}=-\left(c_{M} / r\right) \sin \left(\varepsilon+\lambda_{\text {off }}\right)-\left(c_{T} / r\right) \sin (\varepsilon-\beta)
$$

and expanding the trigonometric sums

$$
\begin{aligned}
\dot{\varepsilon}=- & \left(c_{M} / r\right)\left[\sin \varepsilon \cos \lambda_{\text {off }}+\cos \varepsilon \sin \lambda_{\text {off }}\right] \\
& -\left(c_{T} / r\right)[\sin \varepsilon \cos \beta-\cos \varepsilon \sin \beta]
\end{aligned}
$$

But, by definition $\lambda_{\text {off }}=\sin ^{-1}\left(c_{T} / c_{M} \sin \beta\right)$, and so this can be collapsed somewhat:

$$
\dot{\varepsilon}=(-\sin \varepsilon / r)\left[c_{M} \cos \lambda_{\text {off }}+c_{T} \cos \beta\right]
$$

Again, given the definition of $\lambda_{\text {off }}$ we can show that the statement in brackets is always positive, regardless of $\beta$ :

Because $c_{M}>c_{T}$, we have that $c_{M}^{2}>c_{T}^{2}$, and that

$$
\begin{gathered}
c_{M}^{2}>c_{T}^{2}\left[\sin ^{2} \beta+\cos ^{2} \beta\right], \quad c_{M}^{2}-c_{T}^{2} \sin ^{2} \beta>c_{T}^{2} \cos ^{2} \beta \\
\sqrt{c_{M}^{2}-c_{T}^{2} \sin ^{2} \beta}>\left|c_{T} \cos \beta\right|
\end{gathered}
$$

The term on the left of Eq. (16) is, by simple trigonometry, the first term in the brackets of Eq. (15), which is always positive and, as evidenced in Eq. (16), dominates the second term in magnitude, thus leaving their sum positive.

It is clear from Eq. (15) that $\dot{\varepsilon}$ and $\varepsilon$ are of opposite sign in all cases except $\varepsilon=\pi$ or 0 , where $\dot{\varepsilon}=0$. It follows from this that $\varepsilon$ converges to zero for all initial conditions except $\varepsilon=\pi$.

\section{E. Convergence of Range}

In light of the preceding subsection, we can also prove that the range converges to zero, or, more precisely:

If the angle error gets small enough, the range is strictly decreasing. Specifically, there exists an $\varepsilon_{0}>0$ and a $\delta>0$ such that for any time $t$ at which $|\varepsilon(t)|<\varepsilon_{0}, \dot{r}(t)<-\delta$.

Proof: The dynamics of the range are

$$
\begin{aligned}
\dot{r}(t) & =-c_{M} \cos \lambda-c_{T} \cos \lambda_{T} \\
& =-c_{M} \cos \left(\varepsilon+\lambda_{\text {off }}\right)-c_{T} \cos [\pi-(\varepsilon-\beta)]
\end{aligned}
$$

Suppose that $\varepsilon=0$, then this equation reduces to

$$
\begin{aligned}
\dot{r}(t) & =-c_{M} \cos \lambda_{\text {off }}+c_{T} \cos (-\beta) \\
& =-\left[c_{M} \cos \lambda_{\text {off }}-c_{T} \cos \beta\right] \\
& =:-\delta_{1}
\end{aligned}
$$

where $\delta_{1}$ is a constant, because $\beta$ is constant and is defined by the preceding relation. Note that $\delta_{1}>0$, by the same reasoning as used in the proof of the preceding lemma.

Now, it is obvious that $\dot{r}$ is a continuous function of $\varepsilon$, and so $\dot{r}$ can be made arbitrarily close to $-\delta_{1}$ by choosing $\varepsilon$ sufficiently close to zero. More specifically, if we choose a $\delta$ such that $0<\delta<\delta_{1}$ and set $\delta_{2}=\delta_{1}-\delta$, then

$$
\exists \varepsilon_{0} \quad \text { such that, if } \quad|\varepsilon|<\varepsilon_{0}, \quad \text { then } \quad\left|\dot{r}-\delta_{1}\right|<\delta_{2}
$$

In particular, $\dot{r}<\delta$.

This implies that range will reach zero in finite time.

\section{F. Proof of Perfect Intercept}

In the preceding two subsections we have proved that the angle error converges to zero and that after the angle error reaches a sufficiently small value the range will reach zero in finite time. Now to complete the proof of a perfect intercept we must show that both go to zero simultaneously. That is,

If $\lambda(0)=\varepsilon(0)+\lambda_{\text {off }}$, there exists a time $T$ such that $r(T)=0$, and the following holds:

$$
\lim _{t \rightarrow T} \varepsilon(t)=0
$$

Proof: For the proof of this lemma, our attention returns to the collision triangle in Fig. 4. This represents the paths of the missile and target some short time $\tau$ before collision, short enought that the missile's path can be approximated by a straight line. We show that if, in such a limiting case, $\lambda=\varepsilon+\lambda_{\text {off }}$, then $\varepsilon$ must be zero.

$A$ and $B$ are the positions of missile and target, respectively, at time $t=T-\tau$, and $C$ is the point of impact. The lengths of the lines $A C$ and $B C$ are clearly related to the speeds of the missile and target $c_{M}$ and $c_{T}$, as shown. Also shown are the angles $\lambda$ and $\lambda_{T}$.

Using the sine rule, we can see that

$$
\begin{gathered}
\frac{\sin (\lambda)}{c_{T}(T-\tau)}=\frac{\sin \left(-\lambda_{T}\right)}{c_{M}(T-\tau)}, \quad c_{M} \sin \left(\varepsilon+\lambda_{\text {off }}\right)=-c_{T} \sin (\varepsilon-\beta) \\
\sin \varepsilon\left[c_{M} \cos \lambda_{\text {off }}+c_{T} \cos \beta\right]=0
\end{gathered}
$$

But as we have already shown in the subsection of convergence of angle error, the term in brackets is strictly greater than zero. Therefore, we must have $\sin \varepsilon=0$.

If we consider the case where $\varepsilon=\pi$ and recall from the definition of $\lambda_{\text {off }}$ that $\left|\lambda_{\text {off }}\right|<\pi / 2$, then this means that $|\lambda|>\pi / 2$ because $\lambda=\varepsilon+\lambda_{\text {off }}$. But if $|\lambda|>\pi / 2$, collision could never occur because $c_{M}>c_{T}$. This contradicts the assumption of our lemma, and so $\varepsilon \neq \pi$.

Therefore, the only possible case (modulo $2 \pi$ ) is $\varepsilon=0$, that is, impact angle is perfect. The result is proven by considering the case of $\tau$ going toward zero. 
Corollary 1 follows from the simple substitution of $\left[\varepsilon(t)-\lambda_{\text {off }}\right]$ for $\varepsilon(t)$ in the proof of Theorem 2, which takes us from the first form of the control law to the second, and gives the error described. This really amounts to the desired impact angle being defined differently. The proof of Corollary 2 is simply that the error between $\lambda(t)$ and $\varepsilon(t)+\lambda_{\text {off }}$ for $\mathcal{T}_{1}$, or between $\lambda(t)$ and $\varepsilon(t)$ for $\mathcal{T}_{2}$, decreases exponentially as in the stationary target case.

\section{Comparative Simulation Results}

In this section we compare $\mathrm{CNG}$ to a guidance law in the literature, called biased proportional navigation guidance (BPNG), which aims to solve a similar problem. ${ }^{3}$ The information set required for CNG is strictly smaller than that for BPNG, which requires range information; despite this, CNG performs favorably compared to BPNG. We should note, however, that BPNG is approximately optimal with respect to acceleration energy when the missile starts close to collision course, whereas CNG makes no claims of optimality in this sense.

We use a hybrid guidance law that saturates the values of $\lambda$, which the missile will track. As long as $\left|\varepsilon+\lambda_{\text {off }}\right|$ is greater than a maximum value $\lambda_{\max }$, we instead drive $\lambda$ to be equal to $\pm \lambda_{\max }$. This results in the missile following an arc of a logarithmic spiral (for example, see Ref. 13) centered on the target, during which $\left|\varepsilon+\lambda_{\text {off }}\right|$ is monotonically decreasing. (The logarithmic spiral shape occurs only if the target is stationary; however, $|\varepsilon|$ decreases monotonically in either case.) As soon as it drops below $\lambda_{\max }$, the missile reverts to $\mathrm{CNG}$.

This results in shorter intercept times, but does not effect any of the theoretical results just described. It might be considered as a midcourse phase and a terminal phase, except that the switch is determined by approach angle, not range or flight time.

The following equations define the hybrid law:

$$
\begin{gathered}
u_{c 1}(t)=2 c_{M} \dot{\sigma}(t)+k_{p}\left\{\lambda(t)-\left[\varepsilon(t)+\lambda_{\text {off }}\right]\right\} \\
u_{c 2}(t)=c_{M} \dot{\sigma}(t)+k_{p}\left\{\lambda(t)-\lambda_{\max } \operatorname{sign}\left[\varepsilon(t)+\lambda_{\text {off }}\right]\right\}
\end{gathered}
$$

with the switching rule

$$
u_{c}(t)=\left\{\begin{array}{lll}
u_{c 1}(t) & \text { if } & \left|\varepsilon+\lambda_{\text {off }}\right| \in\left[0, \lambda_{\text {max }}\right) \\
u_{c 2}(t) & \text { if } & \left|\varepsilon+\lambda_{\text {off }}\right| \in\left[\lambda_{\text {max }}, \pi\right]
\end{array}\right.
$$

We used a saturation value of $\lambda_{\max }=60 \mathrm{deg}$.

In our simulations, the missile started $5 \mathrm{~km}$ directly behind the target, and it was required to hit the target with a variety of different impact angles ranging from $\beta=0 \mathrm{deg}$ (tail on) to $170 \mathrm{deg}$ (nearly head on). The missile speed was $c_{M}=1000 \mathrm{~ms}^{-2}$, and the target speed was varied between $c_{T}=50$ and $950 \mathrm{~ms}^{-2}$. The target was maneuvering away from the missile with a angle rate $\dot{\gamma}_{T}=2.3 \mathrm{deg} / \mathrm{s}^{-1}$.

We implemented the guidance law given by Eqs. (7) and (18) in Ref. 3. Gains were chosen as in that paper, but modified for the different missile and target speeds. The gain $k_{p}$ for CNG was $10 c_{M}$.

In the first set of simulations, no saturation of missile acceleration was assumed. In the second set, we limited missile acceleration to $\left|\dot{\gamma}_{M}\right| \leq 40 \mathrm{deg} / \mathrm{s}^{-1}$, as in Ref. 3 .

We found in almost all cases that one of two things would happen. Either 1) the missile would impact the target with extremely small miss distance (essentially zero, subject to simulation accuracy) and very small impact angle error (usually less than one degree); or 2) the missile would fail to impact the target at all. This being the case, we have chosen to plot only the region of successful intercepts, not the actual performance within these regions. A successful intercept was defined as one where the range was less than $5 \mathrm{~m}$ and the angle error less than $5 \mathrm{deg}$, where angle error is calculated as $\left|\sigma-\left(\gamma_{T}+\beta\right)\right|$ at the time when the missile is exactly $10 \mathrm{~m}$ from the target.

The results without any acceleration saturation are plotted in Fig. 7. High-speed targets combined with large impact angles defeated both guidance laws. However, as long as the target speed was less than about two-thirds of the missile speed, any impact angle was achievable with CNG. BPNG was not able to hit the target with

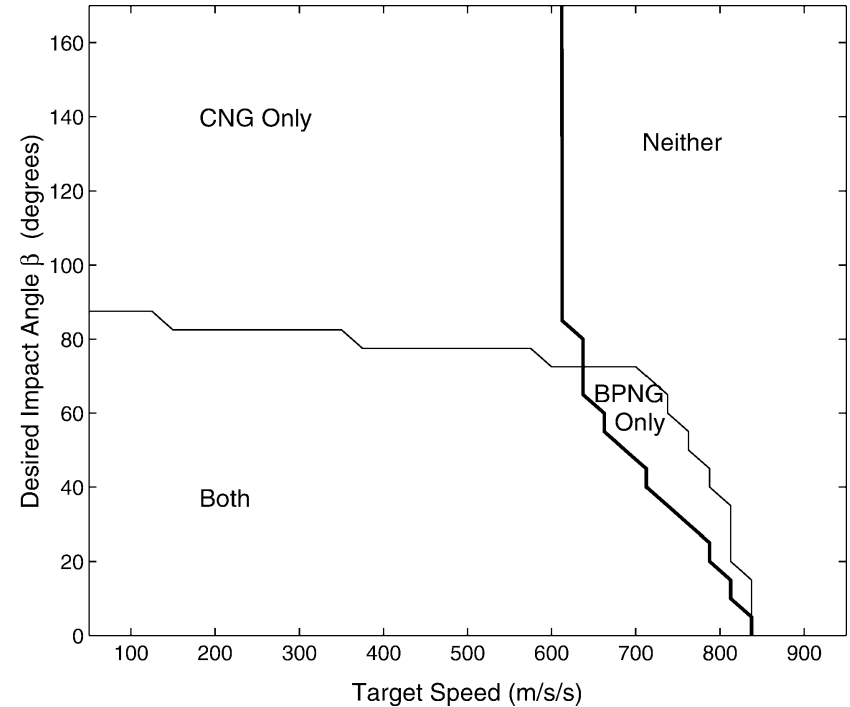

Fig. 7 Envelope of successful intercepts plotted against target speed and desired impact angle.

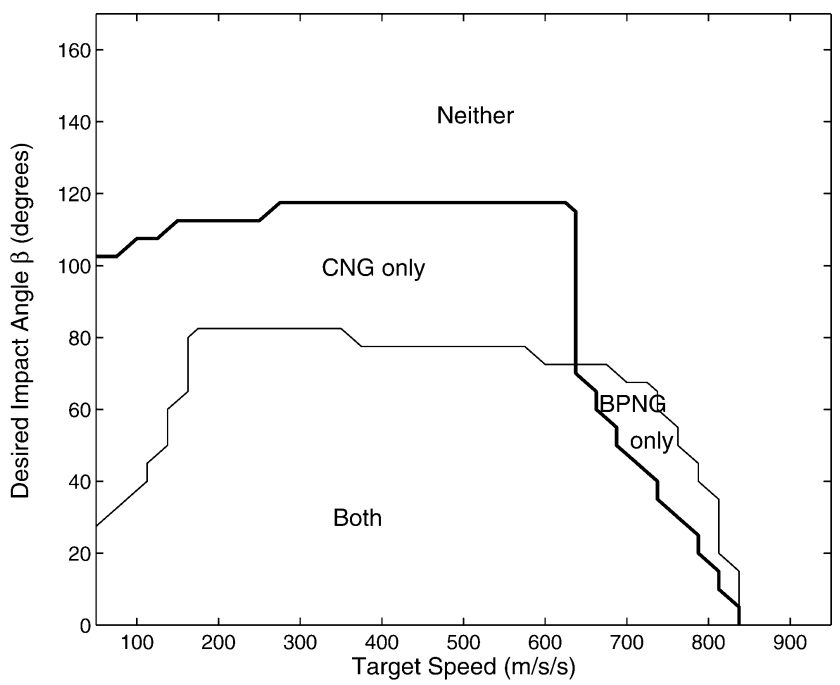

Fig. 8 Envelope of successful intercepts plotted against target speed and desired impact angle, with acceleration saturation.

impact angles wider than 90 deg. For impact angles less than $90 \mathrm{deg}$, BPNG is able to hit slightly faster targets than CNG.

When missile acceleration was limited, the results are somewhat different, as seen in Fig. 8. In this case, both guidance laws were limited in the range of impact angles they could achieve. But again, when the target speed is less than around two-thirds the missile speed, CNG was consistently able to achieve 30-40-deg wider impact angles than BPNG. One again there is a thin sliver of values where BPNG can hit slightly faster targets at narrow impact angles.

In Figs. 9 and 10 we show the trajectories and commanded acceleration for an example with target speed of $100 \mathrm{~m} / \mathrm{s}$ and $\beta=30 \mathrm{deg}$. We can see that BPNG tends to head more directly toward the target early in the intercept and then swing around to the correct approach angle before intercept, whereas CNG tends to take a wider path. As a result, BPNG will have a shorter intercept time; however, it will require a larger acceleration command immediately before impact, as seen in Fig. 10. These effects agree with the results in Figs. 7 and 8 , in which BPNG performed slightly better against faster targets (because of its more direct route to the target) but worse at wider impact angles (because of its need to swing around more sharply at the end of the intercept).

Because CNG attempts to follow a circular path, we can see that over large sections of the intercept, following an initial transient, the commanded acceleration is essentially constant. This might be 


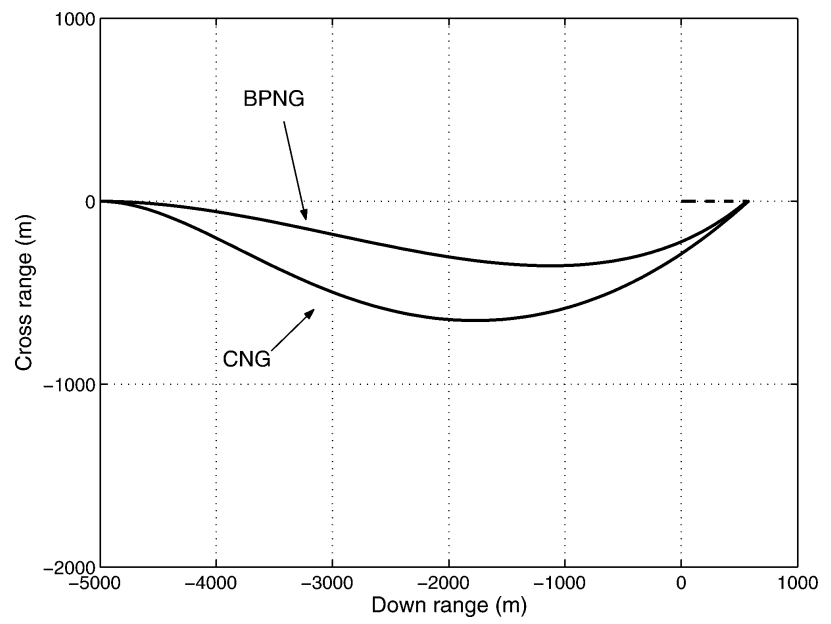

Fig. 9 Example trajectories of a missile guided by CNG and BPNG.

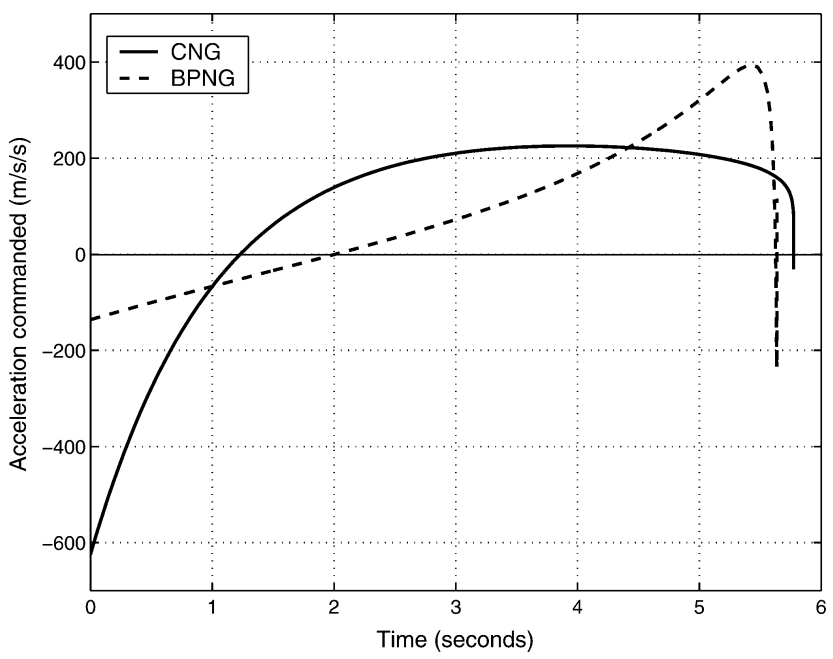

Fig. 10 Commanded acceleration for the trajectories in Fig. 9.

a disadvantage for missile systems that use propulsive means to generate accelerations; thus, $\mathrm{CNG}$ would most likely be better suited to airframes that use aerodynamic means to affect acceleration.

\section{Conclusions}

In this paper, we have considered the problem of precision missile guidance, and presented the circular-navigation-guidance (CNG) law. This law is based on novel and simple, geometric ideas. These ideas were discussed, and some basic theoretical results proved.
Its performance was compared to another law from the literature, and the results are promising. It seems that $\mathrm{CNG}$ is successful over a much wider range of desired impact angles. In a complex field such as missile guidance, there is never one solution that is uniformly better than all others, and for instance our law does not make any claims of optimality with respect to control effort. On the other hand, our law does not require range-to-target information, in constrast to all other impact-angle constrained guidance laws of which the authors are aware.

These considerations make CNG a promising approach for future study and an interesting alternative to current methods of precision missile guidance. Also, these techniques might find fertile application in other areas such as mobile robot control, an area we are currently researching.

\section{Acknowledgment}

This work was supported by the Australian Research Council.

\section{References}

${ }^{1}$ Kim, M., and Grider, K. V., "Terminal Guidance for Impact Attitude Angle Constrained Flight Trajectories," IEEE Transactions on Aerospace and Electronic Systems, Vol. 9, No. 6, 1973, pp. 852-859.

${ }^{2}$ York, R. J., and Pastrick, H. L., "Optimal Terminal Guidance with Constraints at Final Time," Journal of Spacecraft and Rockets, Vol. 14, No. 6, 1977, pp. 381, 382.

${ }^{3}$ Kim, B. S., Lee, G. L., and Han, H. S., "Biased PNG Law for Impact with Angular Constraint," IEEE Transactions on Aerospace and Electronic Systems, Vol. 34, No. 1, 1998, pp. 277-288.

${ }^{4}$ Zarchan, P., Tactical and Strategic Missile Guidance, AIAA, Washington, DC, 1994.

${ }^{5}$ Ben-Asher, J. Z., and Yaesh, I., Advances in Missile Guidance Theory, AIAA, Reston, VA, 1998

${ }^{6}$ Song, T. L., Shin, J. S., and Han, H. S., "Impact Angle Control for Planar Engagements," IEEE Transactions on Aerospace and Electronic Systems, Vol. 35, No. 4, 1999, pp. 1439-1444.

${ }^{7}$ Song, T. L., and Shin, J. S., "Time Optimal Impact Angle Control for Vertical Plane Engagements," IEEE Transactions on Aerospace and Electronic Systems, Vol. 35, No. 2, 1999, pp. 738-742.

${ }^{8}$ Savkin, A. V., Pathirana, P., and Faruqi, F. A., "The Problem of Precision Missile Guidance: LQR and $\mathcal{H}_{\infty}$ Frameworks," IEEE Transactions on Aerospace and Electronic Systems, Vol. 39, No. 3, 2003, pp. 901-910.

${ }^{9}$ Manchester, I. R., and Savkin, A. V., "Circular Navigation Guidance Law with Incomplete Information and Uncertain Autopilot Model," Journal of Guidance, Control, and Dynamics, Vol. 27, No. 8, 2004, pp. 1078-1083.

${ }^{10}$ Petersen, I. R., Ugrinovskii, V. A., and Savkin, A. V., Robust Control Design Using $H^{\infty}$ Methods, Springer-Verlag, London, 2000, Chap. 5.

${ }^{11}$ Petersen, I. R., and Savkin, A. V., Robust Kalman Filtering for Signals and Systems with Large Uncertainties, Birkhauser, Boston, 1999.

${ }^{12}$ Savkin, A. V., and Petersen, I. R., "Recursive State Estimation for Uncertain Systems with an Integral Quadratic Constraint," IEEE Transactions on Automatic Control, Vol. 40, No. 6, 1995, pp. 1080-1083.

${ }^{13}$ Gallier, J., Geometric Methods and Applications: for Computer Science and Engineering, Springer-Verlag, New York, 2001, pp. 435-437. 